3.

\title{
Ueber einen blauen Farbstoff beim Menschen.
}

\section{Von Dr. C. Hennig, Arzt und akademischem Docenten zu Leipzig.}

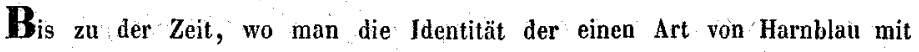
dem vegetabilischen Indigo nachwies, finden sich nur verstreute Beispiele von solchen Erzengnissen; sie sind zum grössten Theile im Archive für pathologische Anatomie und Physiologie zusammengestellt (Bd. I. S. 424.), eines von Billard erzäblt noch Rayer (Bd..III. S, 212 der deutschen Uebersetzung seiner „Darstellong der Hautkrankheiten"). Aus diesen Fällen darf man schon schliessen, dass das pathologische Blau melrere Modificationen hat, wenn sie auch einmal alle in einander übergefülirt werden mögen. Zunächst machen sich zwei Sorten bestimmter kenntlich; eine sei die bewegliche, die andere die beständige oder das wabre Indigo genannt. Die erstere ist vielleicht dieselbe, welche in der von Virchow aufgestellten Stufenreihe vorkommt, welche die Producte der Einwirkung concentrirter Mineralsäuren auf das bis zu einem gewissen Grade ungewandelte Blutroth entlält also vielleicht künstlich nachzaahmen. Hierher gehört wohl Braconnot's Cyanurin. Die letztere, festere Verbindung, ist diejenige, welche offter im Urine (von Spangenberg, krystallinisch zuerst von Virchow), sparsam im Blute (S an son, Lassaigne, Lecanu) und in der Galle (Chevreul), am seltensten, dann aber in grösserer, bisweilen erstaunlicher Menge als Aussonderung der Haut gefunden worden ist. Dem im genannten Archive enthaltenen Falle $(, B$ Büchner fand bei einer 4zjährigen Frau wälirend der Scliwangerschaft Knoten, die nachher indigblaues Pigment absonderten, welches die Wäsche färbte") stelt der Billard'sche zur Seite, wo die massenhafte blaue Hautabsonderung mit verminderter, zeitweis ganz geliemmter Harnaussonderung einherging.

Vor zwei Jahren untersuchte ich die Schïppehen, welche sich auf dem von Pityriasis - hefallenen Theile der behaarten Kopfhaut einer älteren Frauensperson reichlich erzeugten und von blendend weisser Farbe waren. Hier fand sich zwischen den zum Theil feingekörnelten Epidermisstückchen, aber auch in den Haarsäcken, neben amorphem Blutfarbstoff und dukler Körnchenmasse, das im durchgehenden Liclste prachtvoll lasurblane Pigment in rundlichen oder eckigen Stückchen, wie ich es in der medicinisch-chirurgischen Encyklopädie von Prosch und Ploss, Art. „Pityriasis" heschrieben habe. Von Essigsäure und von Aetzkalilauge wurde es nieht angegriffen, Salpetersäure färbte es allmälig grün, dann gelb und brachte es allmälig zum Verschwinden; concentrirte Scliwefelsäure liess es unter abneh. mender Bläue binnen wenigen Minuten verschwinden. - Weder bei diesem Anlasse, noch in einem der folgenden, sah ich die krystallinische Form.

Demnächst habe ich Indigpigment im Urine einiger Kranker, doch unter diesen nur einmal olne vorherige Zugabe von Salz-oder Salpetersäure, und nie in be- 
deutender Menge angetroffen. Der blaue Farbstoff nimmt sich unter dem Mikroskope gerade so aus, wie er oben von der Haut beschriehen wurde, und ist sofort unterscheidbar von jenem, Uroglaucin", welches durch eine Modification des gelben Harnfarbstoffes violett tingirte Harnsäurekrystalle sind.

Endlich fand ich vor Kurzem das mikroskopische Blau an mehreren Theilen des Skelettes eines todtgeborenen Zwillings, dessen Bruder, welcher 10 Tage später starb, jenes Pigment an den nämlichen Stellen nicht aufwies. Beide kamen mit dèm Beckenende voran, und es war jeder Fötus in seinen besonderen Schafliautsack eingeschlossen und hatte seine besondere Placenta. Der von Farbstoff freie stellte sich znerst zur Geburt and wurde durch einfachen Handgriff entwickelt. Er erkrankte einige Tage nach der Geburt, obne Zweifel unter dem Einflusse des Puerperalfiebers seiner Mutter, das von einer umschriebenen Bauchfellentzündung Ursprung genommen, an Entzündung der Unterkieferdrïse linkerseits. Diese Drüse ging rasch in einen Abscess auf, der seiner dicken Wände balber sich nicht nach aussen entleeren konnte; in der Leiche fand sich zugleich Eiter in der rechten Thymushälfte, im rechten Brustfellsacke, am Herzbeutel und auf der Herzbasis; zugleich hänorrhagische Myocarditis. Der zweite Knabe war stärker entwickelt (er wog, obgleiclı 2-3 Wochen zu frülı geboren, 6 Pfund Krämergewicht) und musste aus dem im Eingange verengten Becken mit Mühe, der Kopf mit Itülfe der Zange weggenommen werden. Hatte der Erstgeborene schon eine noch am Todestage bedeutende Intercalation des oberen Randes des Hinterhauptbeines unter die der entsprechenden Scheitelbeine, dazu einen dünnen Bluterguss im Sacke der Spinnwebhaut in der Gegend der Lambdanaht, so war der letztere weit übler davongekommen. Sein linkes Stirnbein hatte am mütterlichen Vorberge einen mebrere Linien tiefen Eindruck erhalten, der sich durch den Fingerdruck nicht wieder von innenher ausgleiclien liess. Auf der dadurcl entstandenen Leiste der Aussenfäche gewabste man 3 der Knickung nicht ganz, aber unter einander parallele, feine Spriinge. Der ganze Knochen war, namentlich gegen die Ränder bin, ungewöhnlich blutreich und an den Bändern etwas dicker als gewöhnlich, Die Verknöcherungspunkte im Brustbeine waren wohl in der richtigen Zahl vorhanden und regelmässig gestellt, aher sehr ungleich fortgeschritten: einige klein, blutarm, doch ziemlich fest; die Mehrzabl aber weit grösser, weicher und von Blut strotzend; auch war der angrenzende Knorpel auf blossen Anblick von Aederchen und Lücken durcluzogen, welche ich am Brustbeine des andern Kindes nicht walurnabm. Auch die Knorpelhaut, namentlich der anstossenden Rippenknorpel, war hyperämisch, dick und haftete fest am Knorpel. Unter dem Mikroskope zeigte sich überwiegende Höhlenbildung; die das sclwammige Gewebe zusammensetzenden Höhlen waren von rother Masse, meist mit von Blut ausgedebnten Gefässen ganz erfüllt, und auch in der angrenzenden Knorpelschiclt gab es ovale Räume, welche nur blassroth oder gelblich gefärbt waren; einer der letzteren liess nur feine Punktmasse als Inhalt unterscheiden. Dazwischen, aher auch noch weiter hinein in den Knorpel gestreut, lagen Stïckchen veränderten Hämatins. Seltener und nur ganz einzeln traf ich - aber sowohl im geknickten Schädellinochen, als auch im Bristbeine und dem Perichondrium deran, 


\section{2}

stossenden Rippenknorpel die schönen Fragmente des blauen Farbstoffes, das grösste von beinahe $\frac{1}{4}$ Par. Linie Durchmesser. Zwischen einem solchen und der kaum $\frac{1}{3}$ Linie davon entfernten Verknöcherungsgrenze bemerkten ich und mehrere Cóllegen, denen ich es zejgte, ein etwa $z_{3}$ Linie langes, mehr verwaschen, als die scharf begrenzten Indigstückchen sich ausnelimendes violet tes Streifchen, so dass an dieser Stelle der Uebergang des Hämatins zum reinen Blau in die Augen fiel. Nach Obigem musste ich annehmen, dass die untersuchten Skeletttheile in krankem Zustande, in dem Falle einer beginnenden, aber angeborenen Rachitis sich befandén. Die dieser Störung zugehörige Blutstauung nun scheint eben the Bedingungen zitr Indigbildung zu enthatten, denn immer traf ich bis jetat das blaue Pigment neben dem amorphen rothen oder gelben und stets in der Nähe von Stasen oder bei allgemeiner Circulationsstörung (Pilyriasis rubra, Knochenhyperänie, Fieberharn).

Als ich diese Sätze in der Märzsitzung der hiesigen medicinischen Gescllschaft vortrug, theilte uns $\mathrm{Hr}$ : Medicinalrath Güntz mit, er habe mit seinem Neffen, deln Assistenzarzte der Irrenanstalt Thonberg bei Leipzig, ,blaue (Indig-) und violette Färbungen in mebreren Gehirnen, besonders bei Hirnerweichung, jüngst auch bei einem- Blödsinnigen gefunden, der an Epilepsie litt. Sie lagerten bald in der grauen, bald in der Marksubstanz und massen $\frac{1}{4}$ his 5 Linien in der Länge bei $\frac{1}{4}$ bis $\frac{1}{3}$ Linie Breite. Die Färbung hielt sich in niederer Temperatur wochenlang."

Erlauben Sie mir, hier eine Stelle aus des jüngeren Gîntz , Dissertatio de anatomia pathologica cerebri vesanorum, Lips. 1853" bier einzuschalten, da sie, unabbängig von meinen Beobachtungen, meine Vermuthung über die Entstelung des blauen Farbstoffes bekräftigt. Es heisst das. Seite 38.:

.... licent afferre, me microscopio adjutum in substantin cerebri medullari Jamjam putredini proxima striam duplitis lineis inclusnm, violace am vidisse, cujus fines praectare serratae fuerunt, cujus membrana autem et circumjecta parvas plicas longitudinales, nonnullis etiani locis rupturas, nullo autem loco nec cellulrs, neque aliud quidquam, quod continuit, ostendit. Brevi jam tempore magis magisque pallidior facta est, In eadem cerebri particula duas alias eodemque colore tinctas animadvert strias, in alius cerebri medulla oblongata denique similem, coeruleo tamen colore, quae brevi tempore palluit, imbutam reperi striam. Quae quidem striae minimorum vasculorum rudimenta, colorem. autem supra commemoratam ha malini vartetales habendas esse, mihi quidem verisimillimum videtur, yuamquam certum hac de re judicium minime ferre velim.

So weit J. M. Th. Güntz.

Endlich ausserte mein Freund Coceius bei einer früheren Gelegenheit, wo joh ibm das Blau aus der Oberhant zeigte, er habe dergleichen Fragmente in gequetschten Muskeln, in der Hornhaut, welche er bei Gehalt an blutfïhrenden Gefässen mit Luft injicirt hatte, in menschlichem Blute, welches in einem Glase eingedampft worden, (in der Galle?) und in menschlichien Excrementen gesehen.

Bemerkenswerth ist tibrigens das vielleicht melr als zufälige stöchiometrische Ergebniss, dass Hämatin nur einer gewöhnilichen Umwandlung, nämlich der Auf- 


\title{
353
}

nahme von 1 Aequiv. Wasser und 3 Sauerstoff bedarf, um die Bestandtheile von Indigblau und benzoësaureın Ammoniak zu enthaJten, denn

Hämatin . . . . . . . . $=\mathrm{C}_{44} \mathrm{H}_{32} \mathrm{~N}_{3} \mathrm{O}_{6} \mathrm{Fe}$

Indigblau, dessen Vorkommen im Harne

Hassal chemisch bestimmt hat, $=\mathrm{C}_{16} \mathrm{H}_{5} \mathrm{~N} \mathrm{O}_{2}$

$$
\begin{aligned}
& \text { Differenz }=\mathrm{C}_{28} \mathrm{H}_{17} \mathrm{~N}_{2} \mathrm{O}_{4} \text { [Fe] } \\
& +0_{3} \cdot a q \\
& =\mathrm{C}_{\mathrm{is}} \mathrm{H}_{18} \mathrm{~N}_{2} \mathrm{O}_{8} \\
& \text { 2. Benzoësäure }=\mathrm{C}_{28} \mathrm{H}_{12} \quad 0_{8}\left[2\left(\mathrm{C}_{14} \mathrm{H}_{5} \mathrm{O}_{3} \cdot \mathrm{aq}\right)\right] \\
& \text { Differenz } \mathrm{H}_{6} \mathrm{~N}_{2}=2 \text { Ammoniak. }
\end{aligned}
$$

Bass aber Sauerstoff aufgenommen werden könne, ist einer Beobachtung von Scherer angemessen, welcher fand, dass Urohämatin unter Aufnabme von 0 zur Abgabe von $\mathrm{CO}_{2}$ gebracht werden könne ( $\mathrm{G}$. Harley in den Verhandlungen der physik,-medic. Ges. in Würzburg. V. 1.).

4.

\section{Ueber reinen Fall von Leukämie.}

\author{
Von Prof. R. Heschl in Krakau.
}

B. Josef, $68 \mathrm{~J}$ ah re alt, Taglölner, wurde im Februar dieses Jahres auf die Klinik des Prof. Hauser in Olmütz wegen einer jauchenden Geschwulst am linken Unterschenkel aufgenommen. Er erinnert sich keiner Krankbeit, ausser mancher kleinen Unpässlichkeiten; seit zwei Jahren jedoch (d. i. 1853) fing er an abzumagern und Trübungen seiner Sinne wahrzunehmen, welche sich allmälig steigerten. Sie bestanden in Kurzsicltigkeit, Funkensehen, Schwerhörigkeit und Ohrensausen, und bei seiner Aufnahme auf die Klinik bestand schon hoch gradige Schwerhörigkeit.

Im August vergangenen Jahres (1854) erlitt er durch einen Baum, den er auf einen Wagen zu laden im Begriffe stand, einen Stoss an die äussere Fläche des linken Unterschenkels, und zwar in dessen unterer Hälfte. Nach einigen Tagen empfand er heftige stechende Sclimerzen an der bezeichneten Stelle, an welcher sich mit Nachlass derselben eine übrigens nicht selhr schmerzhafte Geschwulst zu bilden anfing, welche sich allmälig vergrösserte, in der siebenten Woche nach ibrer Entstehung die Haut durchbrach, sich öffnete und eine geringe Mengo blutiger Flüssigkeit ergossen haben soll. Aus der 0effnung wucherte alsbald eine graurothe mit missfarbiger Oberfläche versehene Geschwulst hervor, welche von dem callös werdenden Hautrande wie eingeschnürt war. Von Zeit zu Zeit hat der Kranke stechende Schmerzen in der Geschwulst, allmälig schwellen die linken Leistendrïsen etwas an.

Archiv f. pathol, Anat. Bd. VIII. Hefl 2 u. 3. 